\title{
Combination of metformin and paclitaxel suppresses proliferation and induces apoptosis of human prostate cancer cells via oxidative stress and targeting the mitochondria-dependent pathway
}

\author{
YUWAN ZHAO*, XIN ZENG ${ }^{*}$, HUANCHENG TANG, DONGCAI YE and JIANJUN LIU \\ Laboratory of Urology, The Affiliated Hospital of Guangdong Medical University, Zhanjiang, Guangdong 524001, P.R. China
}

Received December 6, 2018; Accepted January 22, 2019

DOI: $10.3892 / 01.2019 .10119$

\begin{abstract}
Previous studies have reported that metformin (MET) has anticancer activity. In combination with chemotherapeutic drugs, MET reduces the dosage of chemotherapeutic drugs required and enhances anticancer efficacy. In the present study, the combination of MET and paclitaxel (PTX) in three human prostate cancer (PCa) cell lines (22RV1, PC-3 and $\mathrm{LNCaP}$ ) was evaluated to investigate the effects on proliferation and apoptosis of PCa cells. The present study explored whether their effects were associated with reactive oxygen species (ROS). An MTT assay and microscopy were used to study the effect of MET + PTX on cell growth. Half maximal inhibitory concentration $\left(\mathrm{IC}_{50}\right)$ values were obtained for MET $(12.281 \pm 1.089 \mathrm{mM}$ for $22 \mathrm{RV} 1,2.248 \pm 0.352 \mathrm{mM}$ for PC-3 cells and $3.610 \pm 0.577 \mathrm{mM}$ for LNCaP cells) and PTX $(13.170 \pm 1.12 \mathrm{nM}$ for PC-3 cells) at $48 \mathrm{~h}$. Since the survival rate of 22RV1 and LNCaP cells did not decrease linearly with increasing PTX concentration, it is difficult to estimate accurate $\mathrm{IC}_{50}$; therefore, only $\mathrm{IC}_{50}$ values for PTX in PC- 3 cells were given. When treating the cells with $5 \mathrm{mM}$ MET, the $\mathrm{IC}_{50}$ of PTX decreased to $5.423 \pm 0.734 \mathrm{nM}$ for PC-3 cells. Annexin V and propidium iodide staining was used to investigate apoptosis by flow cytometry. The apoptotic mechanisms of MET + PTX in PCa were investigated by detecting the expression of apoptosis-related proteins, activities of caspase-3/7, intracellular ROS accumulation, mitochondrial membrane potential, and intracellular levels of adenosine 5'-triphosphate (ATP). MET
\end{abstract}

Correspondence to: Dr Jianjun Liu, Laboratory of Urology, The Affiliated Hospital of Guangdong Medical University, 57 Renmin Street South, Zhanjiang, Guangdong 524001, P.R. China

E-mail: jianjunliulab@163.com

*Contributed equally

Abbreviations: MET, metformin; PTX, paclitaxel; NAC, $\mathrm{N}$-acetylcysteine; GSSG, glutathione disulfide; ROS, reactive oxygen species

Key words: prostate cancer, metformin, paclitaxel, apoptosis, reactive oxygen species
+ PTX induced PCa apoptosis and ROS accumulation, and decreased mitochondrial membrane potential and intracellular levels of ATP. Taken together, these results indicated that MET + PTX suppressed PCa cell proliferation in a dose- and time-dependent manner. In addition, MET + PTX induced apoptosis by increasing ROS levels, reducing mitochondrial membrane potential, and activating mitochondrial-dependent apoptotic pathways.

\section{Introduction}

Prostate cancer (PCa) is a malignancy of the urinary system (1). The probability of newly diagnosed $\mathrm{PCa}$ is high in European and American men (2). Worldwide it has become the second leading cause of cancer-associated mortality in men (3). Patients with localized $\mathrm{PCa}$ are generally treated with hormones, surgery, and chemotherapy or radiation therapy. Hormone therapy is effective at early stages; however, a number of patients slowly develop androgen insensitivity $(4,5)$. When advanced PCa develops into castration-resistant $\mathrm{PCa}$ and metastasizes to distant sites, taxanes are often used for treatment (6).

Paclitaxel (PTX) is an alkaloid that has been used as a first-line treatment for $\mathrm{PCa}$ in a clinical setting. It exerts its antitumor effects via cell cycle arrest (7). Resistance to PTX limits its therapeutic effect (8). Chemoresistant metastatic PCa is the most lethal form of cancer in adult men (9); therefore, more effective treatments for $\mathrm{PCa}$ are required.

Metformin (MET) is an oral anti-diabetic drug, commonly used to treat type 2 diabetes mellitus (10). It is the most commonly used biguanide drug, and has a relatively low incidence of toxicity and side effects $(11,12)$. MET has attracted increased attention in recent years due to its possible anticancer activity; it inhibits several tumor types, including PCa (13-15). A number of in vivo experiments have revealed that MET directly affects cancer cell growth. Its effects have been observed in a wide range of cancer cell lines, including PCa cell lines (16,17). MET induces apoptosis and cell cycle arrest, reducing cancer cell growth $(18,19)$. A previous study reported that MET increases sensitivity to chemotherapy and decreases required chemotherapy drug doses in various cancer cell lines (20).

Given its excellent safety profile, low cost and minimal side effects, MET is an attractive candidate as a potential 
anticancer agent. Nevertheless, there remains limited knowledge regarding its anticancer molecular mechanisms. Therefore, the present study investigated the effects of MET in combination with PTX on apoptosis of 22RV1, PC-3 and $\mathrm{LNCaP}$ cells, as well as the molecular mechanisms underlying these effects. In the present study it was demonstrated that MET augmented the effects of PTX.

\section{Materials and methods}

Cell culture. Human PCa cell lines 22RV1, PC-3 and LNCaP were purchased from the Chinese Academy of Sciences Cell Bank (Shanghai, China). The three cell lines were cultured in RPMI-1640 medium (Gibco; Thermo Fisher Scientific, Inc., Waltham, MA, USA), supplemented with $10 \%$ fetal bovine serum (FBS; Gibco; Thermo Fisher Scientific, Inc.) for 22RV1 and PC-3 cells, and with $12 \%$ FBS for LNCaP cells at $37^{\circ} \mathrm{C}$. Finally, a mixture of penicillin and streptomycin (Beyotime Institute of Biotechnology, Shanghai, China) at a final concentration of $1 \%$ was added.

Reagents and antibodies. MET and PTX were purchased from Beijing Solarbio Science \& Technology Co., Ltd. (Beijing, China). MET was dissolved in 1X PBS to a concentration of $2 \mathrm{M}$, and PTX was dissolved in 100\% dimethyl sulfoxide (DMSO) to create a $10 \mathrm{mM}$ stock solution; these were stored at $-20^{\circ} \mathrm{C}$. N-acetylcysteine (NAC) and glutathione disulfide (GSSG) were purchased from Beyotime Institute of Biotechnology. NAC $(100 \mathrm{mM})$ and GSSG $(10 \mathrm{mM})$ in PBS stock solutions were stored at $-20^{\circ} \mathrm{C}$. Antibodies against poly (ADP-ribose) polymerase (PARP; cat. no. 9542), caspase-3 (cat. no. 9665), caspase-9 (cat. no. 9502), B-cell lymphoma 2 (Bcl-2; cat. no. 2872), Bcl-2-associated X protein (Bax; cat. no. 2772), cytochrome $c$ (Cyto-C; cat. no. 11940) and P53 (cat. no. 9284p) were obtained from Cell Signaling Technology, Inc. (Danvers, MA, USA). GAPDH (cat. no. ab37168) antibody was purchased from Abcam (Cambridge, UK). Immunoglobulin G-horseradish peroxidase (IgG-HRP; cat. no. 030181) was purchased from EarthOx Life Sciences (Millbrae, CA, USA).

Cell viability assay. An MTT assay was used to measure cell viability. Briefly, PCa cells, PC-3/LNCaP $\left(4 \times 10^{3}\right.$ cells/well $)$ and 22RV1 ( $1 \times 10^{4}$ cells/well), were seeded in 96-well plates overnight, and were then incubated with various concentrations of MET and PTX at $37^{\circ} \mathrm{C}$ for $6,12,24,48$ and $72 \mathrm{~h}$. MTT $(0.5 \mathrm{mg} / \mathrm{ml})$ was added to each well. After $4 \mathrm{~h}$ of incubation, supernatants were removed and $150 \mu \mathrm{l}$ DMSO was added to each well as a solvent. Using a Multiskan Ascent microplate photometer (EnSpire 2300 Multilabel Reader; PerkinElmer, Inc., Waltham, MA, USA) absorbance was measured at $492 \mathrm{~nm}$. DMSO-treated cells (control group) were regarded as having $100 \%$ viability.

Apoptosis assay. Apoptosis was measured using the Apoptosis Detection kit (BD Pharmingen; BD Biosciences, Franklin Lakes, NJ, USA). Cells ( $1 \times 10^{5}$ cells/well) plated in $6-\mathrm{cm}$ dishes were treated with MET (5 mM) and PTX (10 nM for PC- 3 cells, and $2 \mu \mathrm{M}$ for $22 \mathrm{RV} 1$ and LNCaP cells). After $24 \mathrm{~h}$ of treatment, cells were washed with PBS and harvested. The apoptosis assay was performed according to the manufacturer's

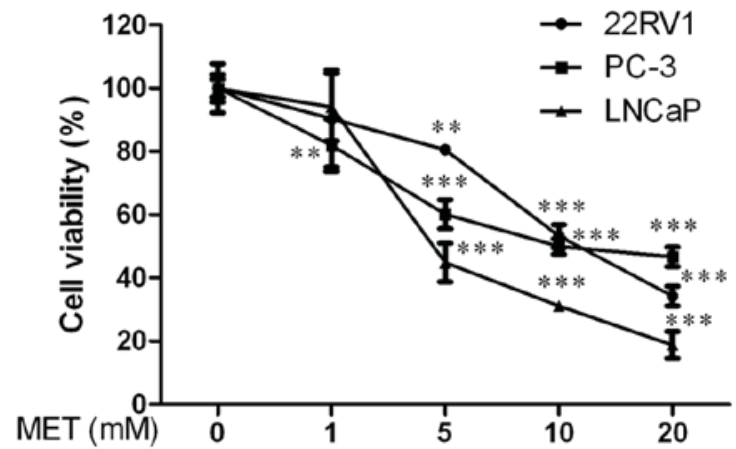

Figure 1. Effects of MET on viability of human prostate cancer cells. Viability of cells following treatment with various concentrations of MET $(0,1,5,10$ and $20 \mathrm{mM})$ was detected by MTT assay at $48 \mathrm{~h}$. Cells treated with DMSO were used as the control group with cell viability set at $100 \%$. ${ }^{* * *} \mathrm{P}<0.01$ and ${ }^{* * * *} \mathrm{P}<0.001$ compared with the control group (DMSO-treated). DMSO, dimethyl sulfoxide; MET, metformin.

protocol using flow cytometry and the results were analyzed using BD FACSDiva 6.1 software (BD Biosciences).

Caspase-Glo 3/7 assays. PC-3 cells, LNCaP cells $\left(4 \times 10^{3}\right.$ cells/well) and $22 \mathrm{RV} 1$ cells $\left(1 \times 10^{4}\right.$ cells/well) were seeded in 96-well plates and exposed to MET and PTX. Equal volumes (100 $\mu$ l) RPMI-1640 medium and caspase-Glo 3/7 reagent (Promega Corporation, Madison, WI, USA) were added to each well, and the cells were incubated for $30 \mathrm{~min}$ at room temperature in the dark. Luminescence was measured by a luminometer (Berthold Sirius L; Titertek-Berthold, Pforzheim, Germany).

Reactive oxygen species (ROS) detection. Cells were pretreated with an antioxidant, NAC (100 $\mu \mathrm{M})$, or a pro-oxidant, GSSG $(5 \mu \mathrm{M})$ for $24 \mathrm{~h}$ prior to the addition of MET. Following treatment with MET ( $5 \mathrm{mM}$ ) and PTX (10 nM for PC-3 cells, and $2 \mu \mathrm{M}$ for $22 \mathrm{RV} 1$ and LNCaP cells) for $12 \mathrm{~h}$, the Reactive Oxygen Species Assay kit (Beyotime Institute of Biotechnology) was used. Cells $\left(1 \times 10^{5}\right.$ cells/well) were collected and resuspended in serum-free medium containing DCFH-DA $(10 \mu \mathrm{M})$. Then cells were incubated at $37^{\circ} \mathrm{C}$ for $20 \mathrm{~min}$ in the dark. ROS levels were measured by flow cytometry and the results were analyzed using BD FACSDiva 6.1 software.

Mitochondrial membrane potential. Cells ( $1 \times 10^{5}$ cells/well) were seeded into 6-well plates overnight, and were subsequently treated with MET (5 mM) and PTX (10 nM for PC-3 cells, and $2 \mu \mathrm{M}$ for $22 \mathrm{RV} 1$ and $\mathrm{LNCaP}$ cells) for $12 \mathrm{~h}$. According to the Mitochondrial Membrane Potential Assay kit (Beyotime Institute of Biotechnology) manufacturer's protocol, the cells were dyed with JC-1 staining fluid and analyzed by flow cytometry and the results were analyzed using BD FACSDiva 6.1 software.

ATP levels. The ATP Assay kit (Beyotime Institute of Biotechnology) was used for detection of ATP levels. Cells $\left(1 \times 10^{5}\right.$ cells/well) were treated with MET $(5 \mathrm{mM})$ and PTX (10 $\mathrm{nM}$ for PC-3 cells, and $2 \mu \mathrm{M}$ for $22 \mathrm{RV} 1$ and LNCaP cells) for $12 \mathrm{~h}$. Subsequently, cells were treated with $200 \mu \mathrm{l}$ lysis buffer (Beyotime Institute of Biotechnology) and collected by 

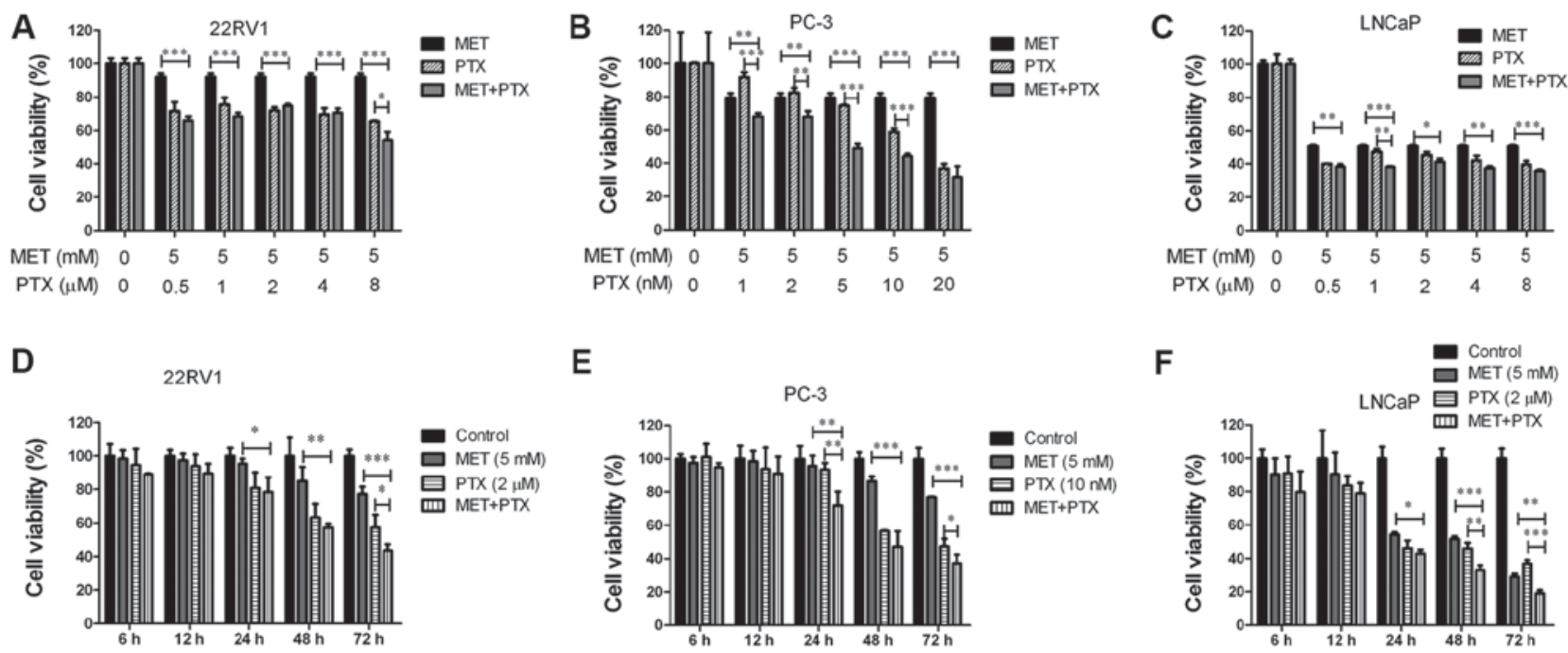

E

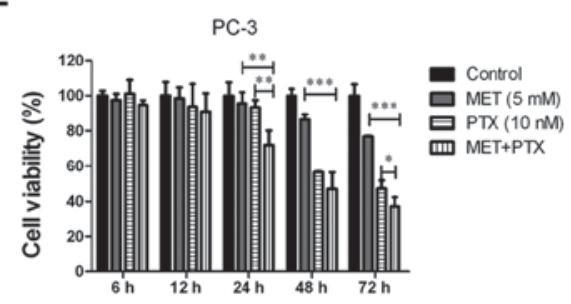

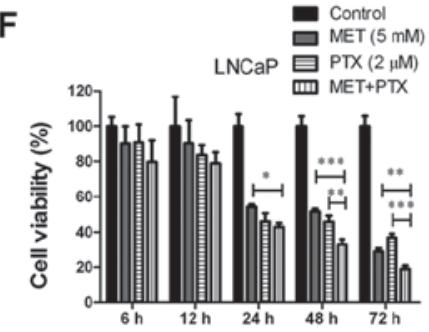

H

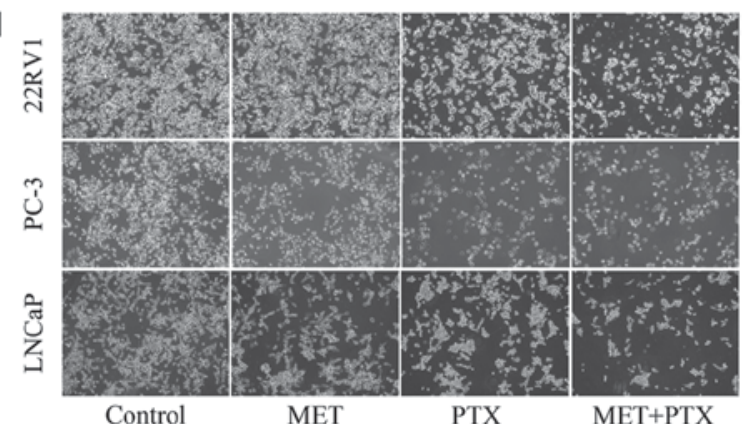

Figure 2. MET in combination with PTX suppresses cell proliferation. (A-C) Prostate cancer cells were treated with MET (5 mM) and PTX (1, 2, 5, 10, 20 nM for PC-3 cells, and $0.5,1,2,4,8 \mu \mathrm{M}$ for $22 \mathrm{RV} 1$ and LNCaP cells) for $48 \mathrm{~h}$, and viability was measured by MTT assay. (D-F) Cell viability was measured by MTT assay following treatment with MET $(5 \mathrm{mM})$ and PTX (10 nM for PC-3 cells, and $2 \mu \mathrm{M}$ for $22 \mathrm{RV} 1$ and LNCaP cells) for $6,12,24,48$ and $72 \mathrm{~h}$. (G) Changes in $\mathrm{IC}_{50}$ of PTX following MET treatment in PC-3 cells. (H) 22RV1, PC-3 and LNCaP cells were treated with MET (5 mM), PTX (10 nM for PC-3 cells, and $2 \mu \mathrm{M}$ for 22RV1 and LNCaP cells) and MET + PTX for $24 \mathrm{~h}$. Images of cells were captured using inverted contrast microscopy (magnification, $\mathrm{x} 100$ ). Cells treated with DMSO were used as the control group with cell viability set at $100 \%$. ${ }^{*} \mathrm{P}<0.05,{ }^{* *} \mathrm{P}<0.01,{ }^{* * *} \mathrm{P}<0.001$. DMSO, dimethyl sulfoxide; $\mathrm{IC}_{50}$, half maximal inhibitory concentration; MET, metformin; PTX, paclitaxel.

centrifugation at $12,000 \mathrm{x}$ for $10 \mathrm{~min}$ at $4^{\circ} \mathrm{C}$. Subsequently, $50 \mu 1$ supernatant and $100 \mu 1$ ATP detection reagent were mixed. Firefly luciferase activity was measured using a luminometer (Berthold Sirius L).

Western blot analysis. Cells were lysed with radioimmunoprecipitation assay buffer (Beyotime Institute of Biotechnology) following MET and PTX treatment for $24 \mathrm{~h}$. Total protein was extracted at $4^{\circ} \mathrm{C}$ and concentration was determined using a bicinchoninic acid assay. Proteins (30 $\mu \mathrm{g}$ protein) were separated by $10 \%$ SDS-PAGE and transferred to a polyvinylidene difluoride membrane (EMD Millipore, Billerica, MA, USA) at $4^{\circ} \mathrm{C}$. The membrane was blocked in $5 \%$ milk in Tris-buffered saline with $1 \%$ Tween-20 at room temperature for $1 \mathrm{~h}$, and incubated with primary antibodies overnight at $4^{\circ} \mathrm{C}$ at the following dilutions: PARP, 1:1,000; caspase-3, 1:1,000; caspase-9, 1:1,000; Bcl-2, 1:1,000; Bax, 1:1,000; Cyto-C, 1:1,000; P53, 1:1,000; and GAPDH, 1:100,000. The membranes were then probed with IgG-HRP antibody (dilution, 1:10,000) for $1 \mathrm{~h}$ at room temperature. Finally, the proteins were detected using Enhanced Chemiluminescent kit (EMD Millipore).

Statistical analysis. The results are presented as the means \pm standard deviation. All experiments were performed in triplicate. Data analysis was performed using a one-way analysis of variance using and the least significant difference post hoc test was used to determine statistical significance. SPSS 16.0 software (SPSS, Inc., Chicago, IL, USA) was used for statistical analysis, and figures were generated using GraphPad Prism 6.0 software (GraphPad Software, Inc., La Jolla, CA, USA). P<0.05, was considered to indicate a statistically significant difference. ImageJ 2.0 software (National Institutes of Health, Bethesda, MD, USA) was used to semi-quantify western blotting images.

\section{Results}

Effect of MET on viability of PCa cells. Firstly, the effect of MET on viability of PCa cells was determined using an MTT assay. 22RV1, PC-3 and LNCaP cells were treated with various concentrations of $\operatorname{MET}(0,1,5,10$ and $20 \mathrm{mM})$ for $48 \mathrm{~h}$. There was a clear reduction in cell viability starting at $5 \mathrm{mM}$ compared with the control group in a dose-dependent manner $(\mathrm{P}<0.01$, Fig. 1). The half maximal inhibitory concentration $\left(\mathrm{IC}_{50}\right)$ value of MET was $12.281 \pm 1.809 \mathrm{mM}$ for $22 \mathrm{RV} 1$ cells, $2.248 \pm 0.352 \mathrm{mM}$ for PC-3 cells and $3.610 \pm 0.557 \mathrm{mM}$ for LNCaP cells at $48 \mathrm{~h}$. These data suggested that MET inhibited the viability of PCa cells in a dose-dependent manner. 
A
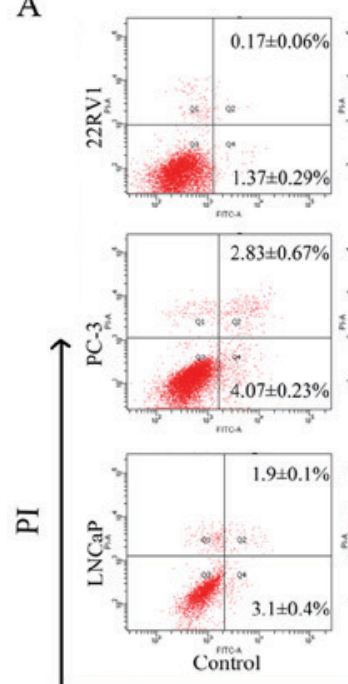

Annexin V
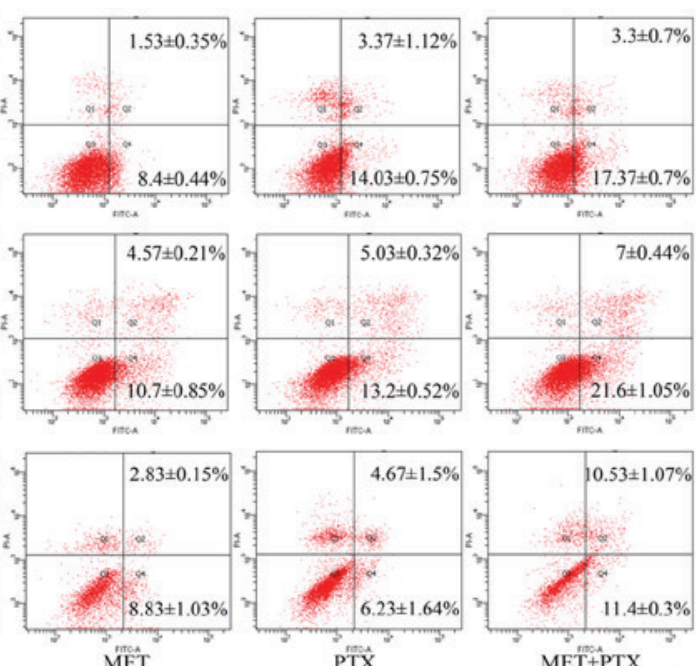

PTX

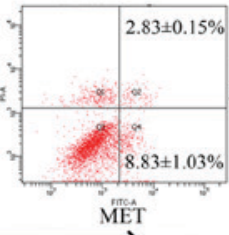

$\stackrel{\mathrm{ME}}{\longrightarrow}$

$\rightarrow$
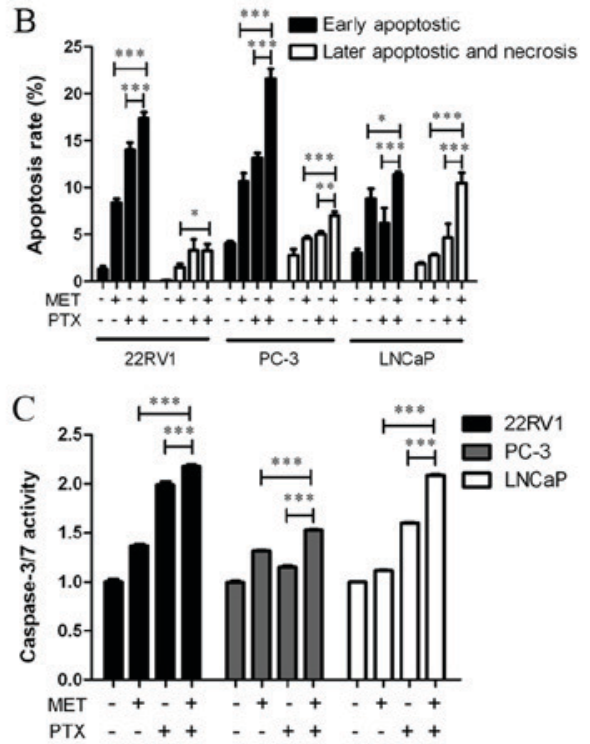

Figure 3. Analysis of MET + PTX-induced apoptosis by Annexin V/PI double-staining and Caspase-Glo 3/7 assays in prostate cancer cells. Cells were treated with MET (5 mM), PTX (10 nM for PC-3 cells, and $2 \mu \mathrm{M}$ for 22RV1 and LNCaP cells) and MET + PTX for $24 \mathrm{~h}$. (A) Flow cytometry was employed to analyze apoptotic cells. (B) Quantification of the percentage of apoptotic cells. (C) Caspase-3/7 activity was detected. ${ }^{*} \mathrm{P}<0.05,{ }^{* *} \mathrm{P}<0.01,{ }^{* * * *} \mathrm{P}<0.001$. MET, metformin; PI, propidium iodide; PTX, paclitaxel.

Effects of MET in combination with PTX on viability of $P C a$ cells. To evaluate whether MET improved the chemosensitivity of PTX, MET and PTX were administered together to PCa cells (Fig. 2). The combination of MET (5 mM) and PTX (1, 2, 5, 10 and $20 \mathrm{nM}$ in PC-3 cells, and 0.5, 1, 2, 4 and $8 \mu \mathrm{M}$ in 22RV1 and LNCaP cells) exhibited a greater inhibitory effect on cell viability (Fig. 2A-C) than MET and PTX did individually. Notably, MET decreased the $\mathrm{IC}_{50}$ of PTX in PC-3 cells (Fig. 2G). These findings suggested that MET inhibited PCa cell proliferation and improved the chemosensitivity of PTX. The concentrations of MET ( $5 \mathrm{mM})$ and PTX (10 nM for PC-3 cells, and $2 \mu \mathrm{M}$ for $22 \mathrm{RV} 1$ and LNCaP cells) were selected to verify that MET and PTX suppressed cell proliferation in a time-dependent manner (Fig. 2D-F). Subsequently, MET and PTX-induced growth inhibition in PCa cells was visualized by microscopy; the cells were treated with MET (5 mM) and PTX (10 $\mathrm{nM}$ for PC-3 cells, and $2 \mu \mathrm{M}$ for 22RV1 and LNCaP cells). Cells cultured without these reagents exhibited characteristic normal growth and shape after $24 \mathrm{~h}$. However, confluence was markedly reduced for cells treated with MET in combination with PTX (Fig. 2H). This finding suggested that MET improved the chemosensitivity of PTX. MET in combination with PTX suppressed cell proliferation in a time-dependent manner.

MET in combination with PTX induces apoptosis of $P C a$ cells. It was also determined whether the augmentation of cell growth inhibition induced by MET in combination with PTX was associated with an increase in apoptosis of PCa cells. The cells were treated with MET $(5 \mathrm{mM})$ and PTX (10 nM for PC- 3 cells, and $2 \mu \mathrm{M}$ for $22 \mathrm{RV} 1$ and $\mathrm{LNCaP}$ cells). After $24 \mathrm{~h}$ of treatment, cells were labeled with Annexin V-fluorescein isothiocyanate/propidium iodide (PI) and analyzed by flow cytometry. The apoptotic effect of MET + PTX was much greater than in the single drug groups (Fig. 3A and B). This suggested that MET in combination with PTX significantly induced early and late apoptosis of PCa cells.
A caspase-3/7 activity assay was used, as shown in Fig. 3C. MET in combination with PTX markedly increased the activity of caspase-3/7. In addition, the expression levels of apoptosis-associated proteins (PARP, P53, Bcl-2, Bax, Cyto-C, caspase-3 and caspase-9) were measured using western blotting. Caspase family members are key proteins in apoptosis (21). MET in combination with PTX markedly increased the expression levels of cleaved caspase-3/9, Bax, P53, Cyto-C and PARP. However, it was identified that treatment with MET and PTX significantly decreased Bcl-2 expression, compared with levels in the single drug groups (Fig. 4A and B). As PC-3 cells in the control group had undetectable expression of Clv-PARP and Cyto-C, it was not possible to present quantification of the increase in expression in relation to control expression in these cells.

MET induces growth suppression and apoptosis of PCa cells via the production of ROS. An MTT assay and a Reactive Oxygen Species Assay kit were used to determine whether MET induced growth suppression and apoptosis via elevation of intracellular ROS levels. Cells were pretreated with an antioxidant, NAC $(100 \mu \mathrm{M})$, or a prooxidant, GSSG $(5 \mu \mathrm{M})$, for $24 \mathrm{~h}$ prior to the addition of MET. NAC and GSSG in the cells were then removed and MET was added for another $24 \mathrm{~h}$. NAC blocked MET-induced cell growth suppression (Fig. 5A) and increased ROS levels (Fig. 5B). GSSG augmented MET-induced cell growth inhibition and promoted ROS production. This suggested that MET inhibited the growth of PCa cells via the production of ROS.

MET in combination with PTX suppresses cell growth and induces apoptosis by increasing ROS production, decreasing mitochondrial membrane potential and decreasing ATP levels in PCa cells. 22RV1, PC-3 and LNCaP cells were treated with MET $(5 \mathrm{mM})$ in combination with PTX (10 $\mathrm{nM}$ in PC-3 cells, $2 \mu \mathrm{M}$ in $22 \mathrm{RV} 1$ and $\mathrm{LNCaP}$ cells) for $12 \mathrm{~h}$, and ROS 


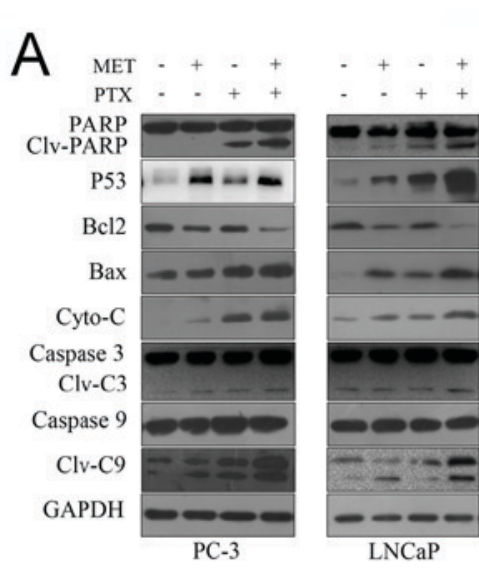

Bax
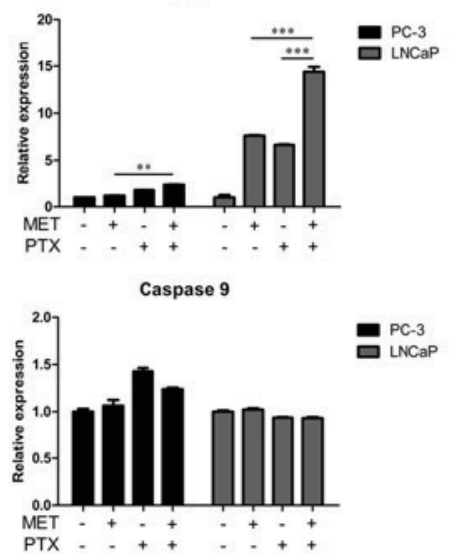
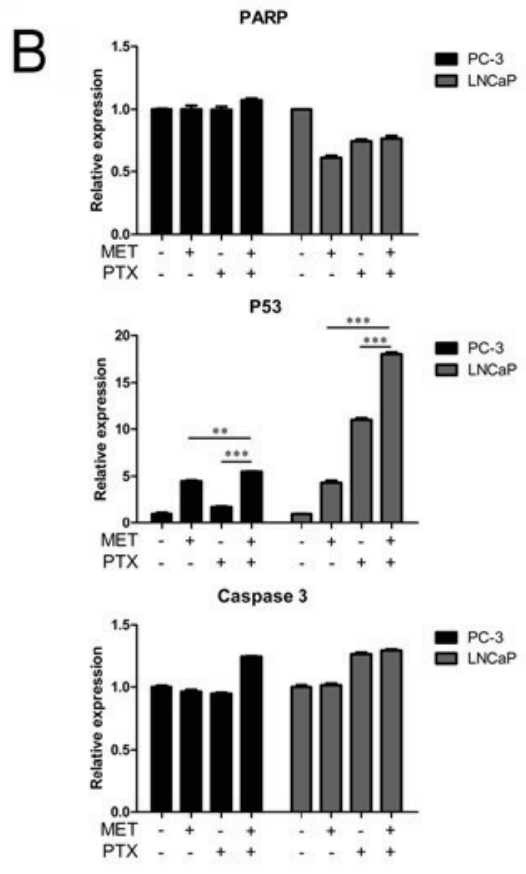

Clv-C9

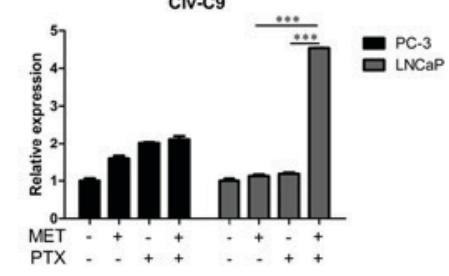

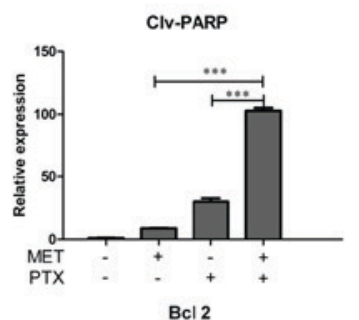

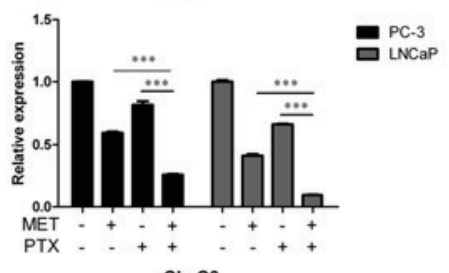

Clv-C3

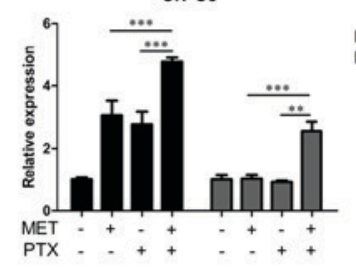

Cyto-c

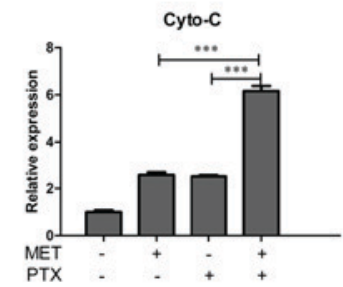

Figure 4. Western blot analysis of apoptosis-associated proteins in prostate cancer cells. Cells were treated with MET (5 mM), PTX (10 nM for PC-3 cells, and $2 \mu \mathrm{M}$ for LNCaP cells) and MET + PTX for $24 \mathrm{~h}$. (A) Western blot analysis was used to detect the expression of apoptosis-associated proteins. (B) Semi-quantification of western blotting. ${ }^{* *} \mathrm{P}<0.01,{ }^{* * *} \mathrm{P}<0.001$. Bax, Bcl-2-associated X protein; Bcl-2, B-cell lymphoma 2; C3, caspase-3; C9, caspase-9; Clv, cleaved; Cyto-C, cytochrome $c$; MET, metformin; PARP, poly (ADP-ribose) polymerase; PTX, paclitaxel.

production was measured. MET + PTX significantly increased the production of ROS in LNCaP cells (Fig. 6A and B). The imbalance of ROS may promote mitochondrial dysfunction and lead to mitochondria-mediated apoptosis. To evaluate the dysfunction in mitochondrial energy production, the mitochondrial membrane potential was measured. It is known that mitochondrial damage during apoptosis alters the mitochondrial membrane potential and intracellular levels of ATP in PCa cells. The present study identified that the mitochondrial membrane potential (Fig. 6C and D) and intracellular levels of ATP (Fig. 6E) were decreased by MET + PTX, and levels were significantly decreased in the MET + PTX group compared with levels in the single drug groups. This suggested that apoptosis of PCa cells mediated by MET + PTX was associated with damage to the mitochondrial membrane.

\section{Discussion}

Chemotherapeutic regimens are commonly used to inhibit tumor growth; nevertheless, these often have side effects. Chemotherapy drugs not only have side effects but cancer cells also often develop resistance to chemotherapeutic agents, limiting their efficacy. Previously, MET was identified as an attractive anticancer adjuvant drug combined with chemotherapeutic drugs, which may improve treatment efficacy and lower the dose of chemotherapeutic agents required.
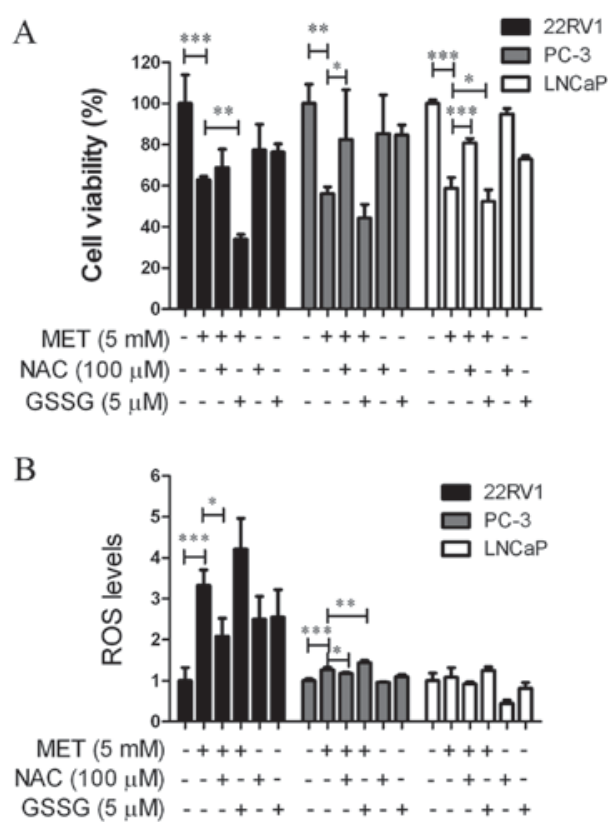

Figure 5. MET inhibits cell viability and increases production of ROS Prostate cancer cells were treated with MET $(5 \mathrm{mM})$ and antioxidant NAC $(100 \mu \mathrm{M})$ or prooxidant GSSG $(5 \mu \mathrm{M})$ for $24 \mathrm{~h}$. (A) Viability was detected by MTT assay. (B) ROS levels were detected using a Mitochondrial Membrane Potential Assay kit. ${ }^{*} \mathrm{P}<0.05,{ }^{* *} \mathrm{P}<0.01,{ }^{* * *} \mathrm{P}<0.001$. GSSG, oxidized glutathione; MET, metformin; NAC, N-acetylcysteine; ROS, reactive oxygen species. 

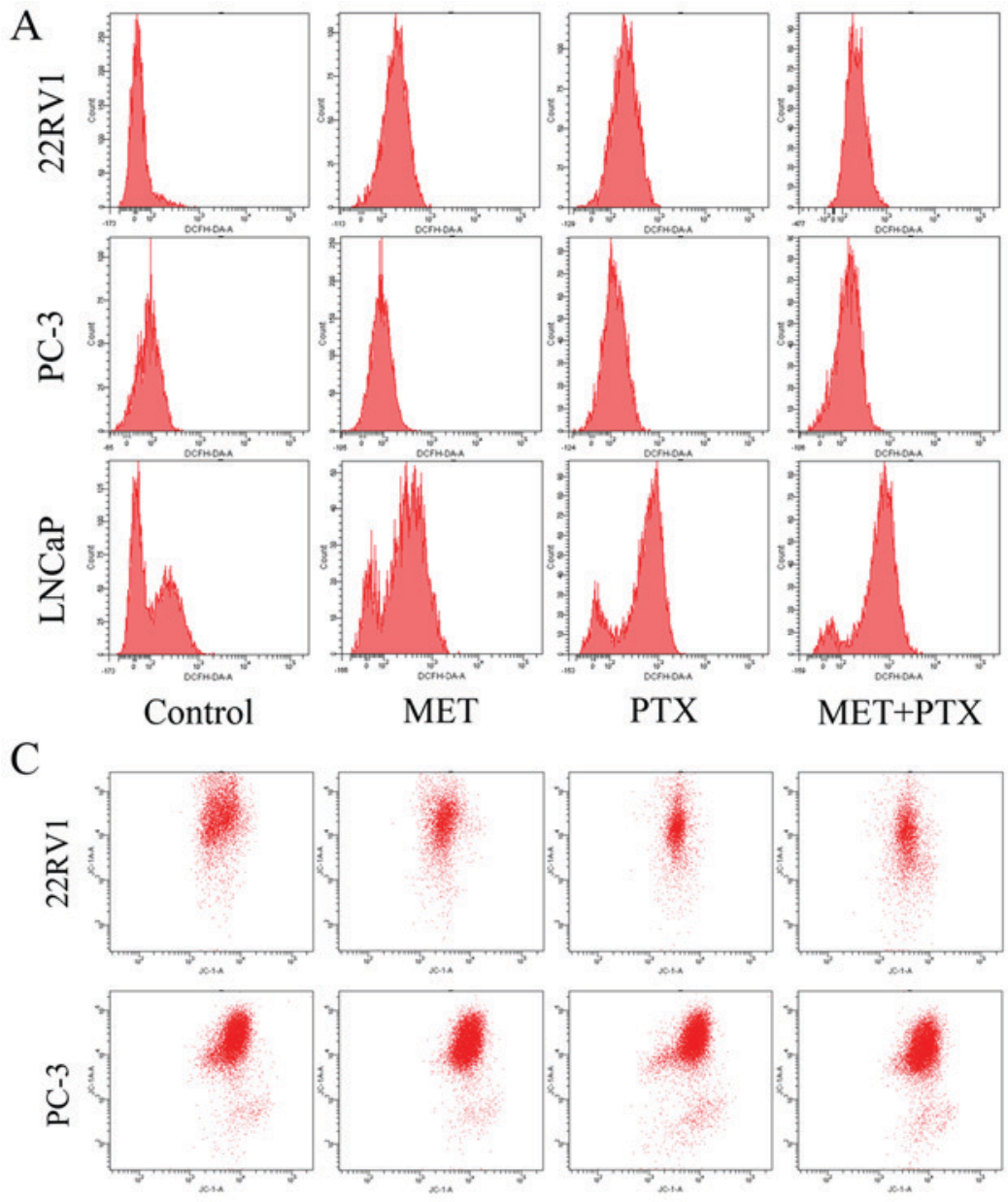

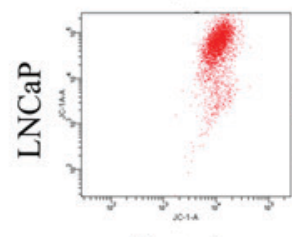

Control

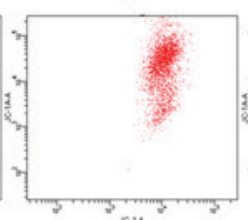

MET

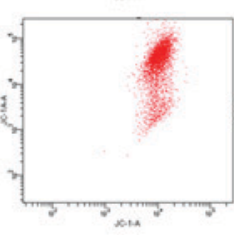

PTX

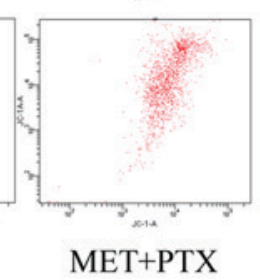

B

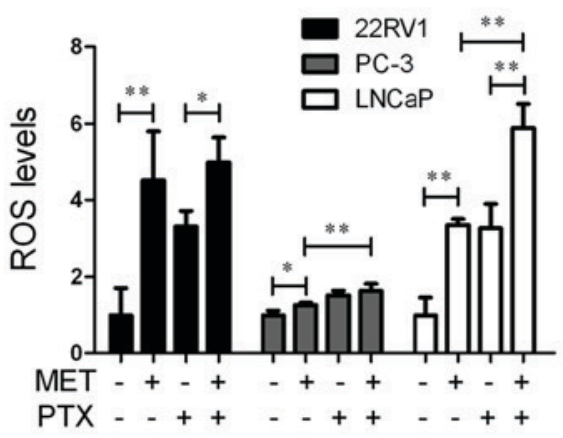

$\mathrm{D}$

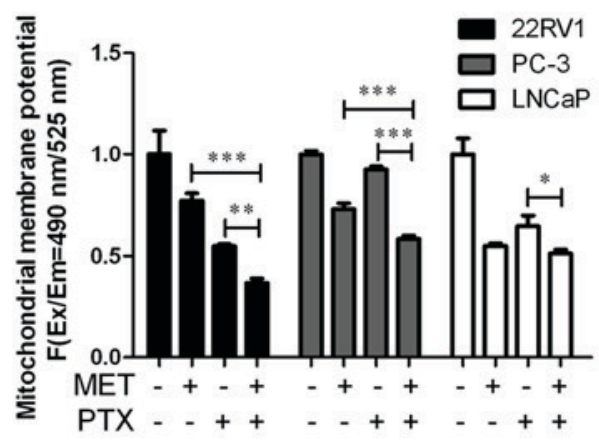

E

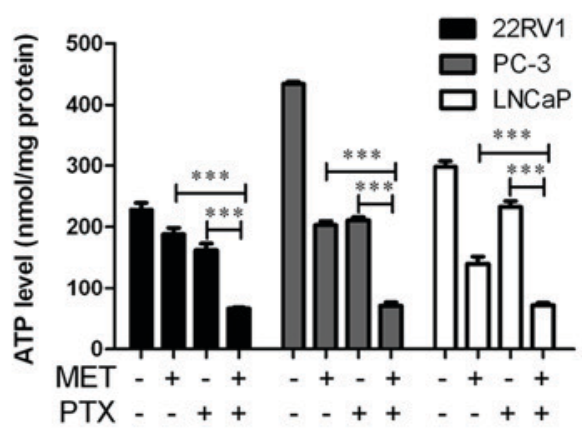

Figure 6. MET in combination with PTX increases ROS production, decreases mitochondrial membrane potential and decreases ATP levels in prostate cancer cells. Cells were treated with MET ( $5 \mathrm{mM}$ ) in combination with PTX (10 nM in PC-3 cells, and $2 \mu \mathrm{M}$ in $22 \mathrm{RV} 1$ and LNCaP cells) for $12 \mathrm{~h}$. (A and B) Intracellular level of total ROS was detected. (C and D) Cells were stained with JC-1 dye and analyzed by flow cytometry. (E) ATP production was detected. * $<0.05$, ${ }^{* *} \mathrm{P}<0.01,{ }^{* * *} \mathrm{P}<0.001$. MET, metformin; PTX, paclitaxel; ROS, reactive oxygen species.

In the present study, the antitumor activity of MET + PTX was evaluated in PCa cells. MET exhibited potential growth inhibitory activity against PCa cells, as determined using the MTT assay. MET and PTX exhibited enhanced ability to reduce tumor proliferation and growth. It was demonstrated that PTX and MET, on their own or in combination, exhibited anti-proliferative effects against cultured PCa cell lines in a time- and dose-dependent manner. However, there were differential sensitivities, in terms of effectiveness of the treatment dosages, among the cell lines. $\mathrm{LNCaP}$ is an early stage androgen-dependent PCa cell line. whereas 22RV1 and PC-3 are androgen-independent PCa cell lines. PC-3 cells have no androgen receptor and exhibit moderate metastatic potential. Therefore, the cell lines exhibit different sensitivity to drugs.

A number of reports have suggested that chemotherapeutic agents exert anti-proliferative effects by inducing apoptosis. It was observed that MET-treated cells exhibit reduced levels of ROS-mediated matrix membrane potential (22). Mitochondria serve a key role in ROS production (23); the present data suggested that MET + PTX induced apoptosis via increasing intracellular ROS levels, and reducing mitochondrial membrane potential and ATP. Notably, an increase in ROS serves a role in the effect of MET + PTX on PCa cells. In the future, we aim to explore whether antioxidant pretreatment can inhibit the effect of MET + PTX on cell proliferation and apoptosis. In the present study, antioxidant NAC and prooxidant GSSG were used to verify that MET increased intracellular ROS levels in PCa cells. NAC pretreatment led to a decrease in MET-mediated production of ROS in 22RV1 and PC-3 compared to MET treatment alone, and had no effect in LNCaP cells. GSSG had no effect on ROS levels in PC-3 and LNCaP cells compared with MET group. In the present study, antioxidant NAC attenuated and prooxidant GSSG increased the effect of MET on ROS production in PCa cells. As is 


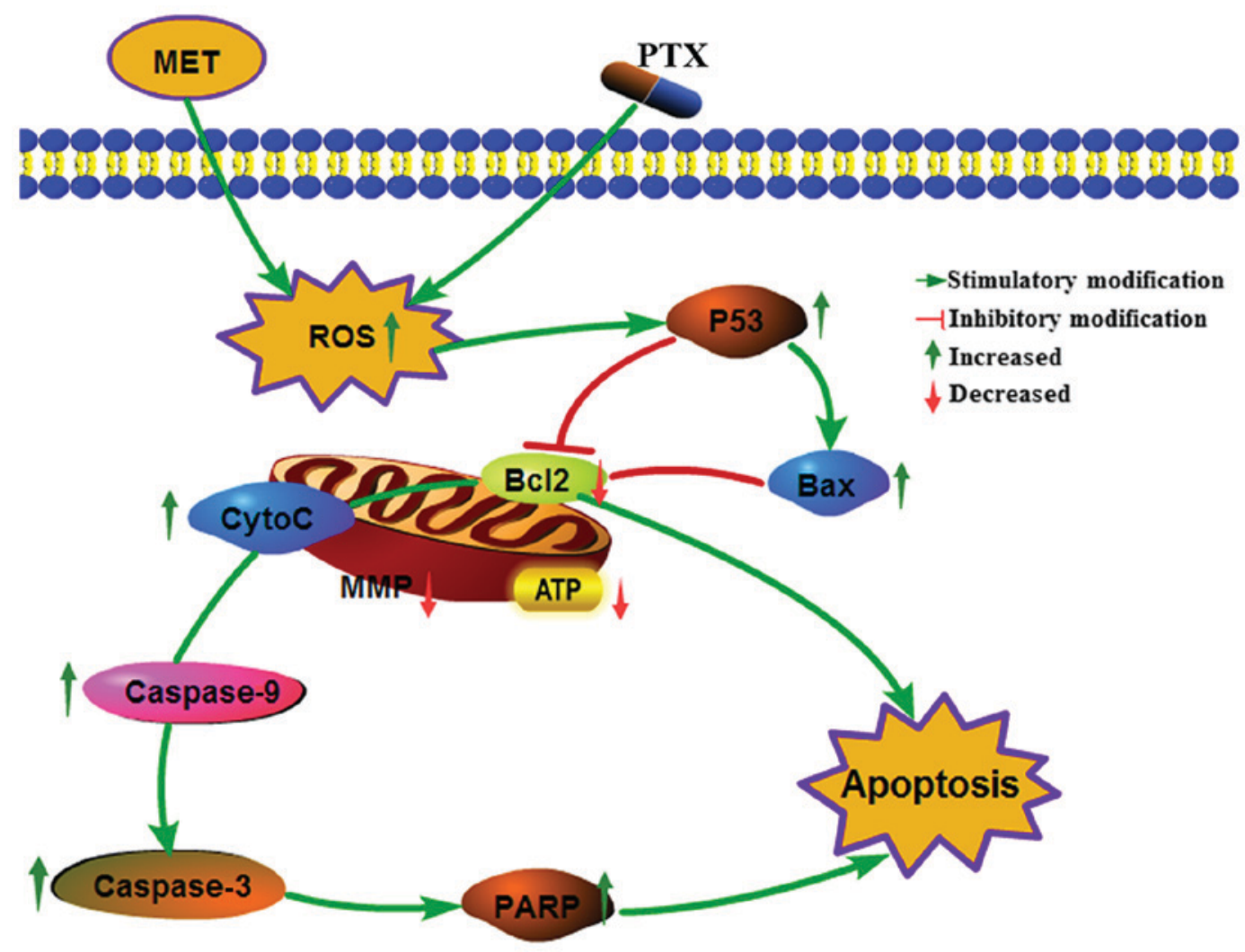

Figure 7. Cellular pathway of the effects of MET + PTX-induced growth inhibition and apoptosis of PCa cells. MET + PTX increased oxidative stress, and decreased MMP and ATP levels in PCa cells. MET + PTX upregulated the production of P53, PARP, capase3/9, Bax, Cyto-C, and downregulated the production of Bcl-2, promoting the release of Cyto-C, increasing caspase-3/7 activities, and potentiating apoptosis in PCa cells. Bax, Bcl-2-associated X protein; Bcl-2, B-cell lymphoma 2; CytoC, cytochrome $c$; MET, metformin; MMP, mitochondrial membrane potential; PARP, poly (ADP-ribose) polymerase; PCa, prostate cancer; PTX, paclitaxel.

commonly known, the androgen receptor serves an important role in the development of PCa. LNCaP is an early stage androgen-dependent growth PCa cell line, PC-3 cells have no androgen receptor, and 22RV1 is an androgen-independent growth PCa cell line. A previous study demonstrated that physiological stimulation of the androgen receptor increases ROS production (24). The androgen receptor may be an important target to investigate differences among the three studied cell lines concerning ROS levels in response to NAC and GSSG. Therefore, we aim to further investigate whether the androgen receptor is involved in the effect of antioxidant NAC and prooxidant GSSG on ROS levels in PC-3, 22RV1 and LNCaP cells.

Increasing intracellular ROS, and reducing mitochondrial membrane potential and ATP induces mitochondrial damage via Cyto-C release from the mitochondria, which in turn activates downstream caspase activity. Bcl-2 family proteins (Bcl-2, Bax and $\mathrm{Bcl}-2$ homologous antagonist killer) participate in the apoptotic pathway leading to cell death. PTX sensitivity is determined by the anti-apoptotic protein Bcl-extra large $(25,26)$. PTX downregulates Bcl-2 and activates caspases and PARP $(27,28)$, resulting in induction of apoptosis. Annexin V and PI staining and analysis by flow cytometry demonstrated that MET + PTX induced PCa cell apoptosis. Finally, the effects of MET + PTX on the expression levels of various proteins, including caspase-3/9, Bax, Bcl2, PARP, Cyto-C and P53, were studied by western blot analysis.
MET + PTX-treated cells exhibited decreased expression of Bcl-2 protein, and increased expression of caspase-3/9, Bax, PARP, Cyto-C and P53 proteins. Taken together, these data suggested that MET + PTX suppressed proliferation and induced apoptosis of human PCa cells via ROS, promoting expression of the pro-apoptotic protein P53, and inducing mitochondrial damage. P53 promoted expression of Bax, inhibited expression of $\mathrm{Bcl}-2$ and mitochondrial damage, and $\mathrm{Bcl}-2$ promoted Cyto-C release from mitochondria. This resulted in the activation of caspase-dependent apoptotic pathways (Fig. 7). A limitation of the present study is that it was limited to in vitro data; therefore, an in vivo study will be seriously considered in the future.

In conclusion, this study demonstrated that MET combined with PTX suppressed cell growth and induced apoptosis of PCa cells via mitochondria-mediated apoptotic pathways. These findings provide promising insights into novel, potential therapeutic strategies for $\mathrm{PCa}$.

\section{Acknowledgements}

Not applicable.

\section{Funding}

This study was funded by The National Natural Science Funds of China (grant no. 81272833). 


\section{Availability of data and materials}

The datasets used and/or analyzed during the current study are available from the corresponding author on reasonable request.

\section{Authors' contributions}

YZ and JL designed the experiments. YZ, XZ, HT and DY performed the experiments. $\mathrm{YZ}$ and $\mathrm{XZ}$ participated in data and statistical analyses. YZ and JL wrote the article and prepared figures. JL provided the financial support. All authors read and approved the final manuscript.

\section{Ethics approval and consent to participate}

Not applicable.

\section{Patient consent for publication}

Not applicable.

\section{Competing interests}

The authors declare that they have no competing interests.

\section{References}

1. Yang Y and $\mathrm{Wu} \mathrm{XH}$ : Study on the influence of metformin on castration-resistant prostate cancer PC-3 cell line biological behavior by its inhibition on PLC $\varepsilon$ gene-mediated Notch1/Hes and androgen receptor signaling pathway. Eur Rev Med Pharmaco Sci 21: 1918-1923, 2017.

2. Crawford ED: Epidemiology of prostate cancer. Urology 62: 3-12, 2003

3. Siegel RL, Miller KD and Jemal A: Cancer statistics, 2015: CA Cancer J Clin 65: 5-29, 2015.

4. Sandblom G and Varenhorst E: Incidence rate and management of prostate carcinoma. Biomed Pharmacother 55: 135-143, 2001.

5. Zitzmann S, Mier W, Schad A, Kinscherf R, Askoxylakis V, Kramer S, Altmann A, Eisenhut $M$ and Haberkorn U: A new prostate carcinoma binding peptide (DUP-1) for tumor imaging and therapy. Clin Cancer Res 11: 139-146, 2005.

6. Chi K, Hotte SJ, Joshua AM, North S, Wyatt AW, Collins LL and Saad F: Treatment of mCRPC in the AR-axis-targeted therapy-resistant state. Ann Oncol 26: 2044-2056, 2015.

7. Weaver BA: How Taxol/paclitacel kills cancer cells. Mol Biol Cell 25: 2677-2681, 2014.

8. Sobue S, Mizutani N, Aoyama Y, Kawamoto Y, Suzuki M, Nozawa Y, Ichihara M and Murate T: Mechanism of paclitaxel resistance in a human prostate cancer cell line, PC3-PR, and its sensitization by cabazitaxel. Biochem Biophys Res Commun 479: 808-813, 2016.

9. Yang Y, Ma Y, Sheng J, Huang Y, Zhao Y, Fang W, Hong S, Tian Y, Xue C and Zhang L: A multicenter, retrospective epidemiologic survey of the clinical features and management of bone metastatic disease in China. Chin J Cancer 35: 40, 2016.

10. Hatoum D and McGowan EM: Recent advances in the use of metformin: Can treating diabetes prevent breast cancer? Biomed Res Int 2015: 548436, 2015.
11. Dowling RJ, Goodwin PJ and Stambolic V: Understanding the benefit of metformin use in cancer treatment. BMC Med 9: 33, 2011.

12. Gou S, Cui P, Li X, Shi P, Liu T and Wang C: Low concentrations of metformin selectively inhibit $\mathrm{CD} 133^{+}$cell proliferation in pancreatic cancer and have anticancer action. PLoS One 8: e63969, 2013.

13. Noto H, Goto A, Tsujimoto $\mathrm{T}$ and Noda M: Cancer risk in diabetic patients treated with metformin: A systematic review and meta-analysis. PLoS One 7: e33411, 2012.

14. Pollak MN: Investigating metformin for cancer prevention and treatment: The end of the beginning. Cancer Discov 2: 778-790, 2012.

15. Del Barco S, Vazquez-Martin A, Cufí S, Oliveras-Ferraros C, Bosch-Barrera J, Joven J, Martin-Castillo B and Menendez JA: Metformin: Multi-faceted protection against cancer. Oncotarget 2: 896-917, 2011.

16. Zakikhani M, Dowling R, Fantus IG, Sonenberg N and Pollak M: metformin Is an AMP kinase-dependent growth inhibitor for breast cancer cells. Cancer Res 66: 10269-10273, 2006.

17. Goodwin PJ, Ligibel JA and Stambolic V: Metformin in breast cancer: Time for action. J Clin Oncol 27: 3271-3273, 2009.

18. Wang LW, Li ZS, Zou DW, Jin ZD, Gao J and Xu GM: metformin induces apoptosis of pancreatic cancer cells. World J Gastroenterol 14: 7192-7198, 2008.

19. Shank JJ, Yang K, Ghannam J, Cabrera L, Johnston CJ, Reynolds RK and Buckanovich RJ: Metformin targets ovarian cancer stem cells in vitro and in vivo. Gynecol Oncol 127: 390-397, 2012

20. Iliopoulos D, Hirsch HA and Struhl K: Metformin decreases the dose of chemotherapy for prolonging tumor remission in mouse xenografts involving multiple cancer cell types. Cancer Res 71: 3196-3201, 2011.

21. Abedi H, Aghaei M, Panjehpour $M$ and Hajiahmadi S: Mitochondrial and caspase pathways are involved in the induction of apoptosis by IB-MECA in ovarian cancer cell lines. Tumor Biol 35: 11027-11039, 2014.

22. Cheng $G$ and Lanza-Jacoby S: Metformin decreases growth of pancreatic cancer cells by decreasing reactive oxygen species: Role of NOX4. Biochem Biophys Res Commun 465: 41-46, 2015.

23. Ding H, Han C, Guo D, Chin YW, Ding Y, Kinghorn AD and D'Ambrosio SM: Selective induction of apoptosis of human oral cancer cell lines by avocado extracts via a ROS-mediated mechanism. Nutr Cancer 61: 348-356, 2009.

24. Khandrika L, Kumar B, Koul S, Maroni P, and Koul HK: Role of Oxidative Stress in Prostate Cancer. Cancer Lett 282: 125-136, 2009.

25. Harley ME, Allan LA, Sanderson HS and Clarke PR: Phosphorylation of Mcl-1 by CDK1-cyclin B1 initiates its Cdc20-dependent destruction during mitotic arrest. EMBO J 29: 2407-2420, 2010.

26. Castilla C, Flores ML, Medina R, Perez-Valderrama B, Romero F, Tortolero M, Japón MA and Sáez C: Prostate cancer cell response to paclitaxel is affected by abnormally expressed securin PTTG1. Mol Cancer Ther 13: 2372-2383, 2014.

27. Tudor G, Aguilera A, Halverson DO, Laing ND and Sausville EA: Susceptibility to drug-induced apoptosis correlates with differential modulation of Bad, Bcl-2 and Bcl-xL protein levels. Cell Death Differ 7: 574-586, 2000

28. Lim SJ, Choi MK, Kim MJ and Kim JK: Alpha-tocopheryl succinate potentiates the paclitaxel-induced apoptosis through enforced caspase 8 activation in human H460 lung cancer cells. Exp Mol Med 41: 737-745, 2009.

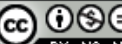

This work is licensed under a Creative Commons Attribution-NonCommercial-NoDerivatives 4.0 International (CC BY-NC-ND 4.0) License. 